\title{
ON A PROBLEM ABOUT CYCLIC SUBGROUPS OF FINITE GROUPS
}

\author{
by OSCAR E. BARRIGA \\ (Received 16th May 1975)
}

\section{Introduction}

Let $G$ be a finite group and let $S$ be a subgroup of $G$ with core $(S)=$ $\bigcap_{x \in G} x^{-1} S x=1$.

We say that $(G, S)$ has property (*) if there exists $x \in G$ such that $S \cap x^{-1} S x=1$.

Conditions on $G, S$ which ensure that $(G, S)$ has property (*) have been found by N. Ito (4), J. S. Broadkey (2), M. Herzog (3) and T. J. Laffey (5). In particular, T. J. Laffey has considered the case where $S$ is cyclic and has shown that $(G, S)$ has property $(*)$ if $G$ is simple and conjectured that $(G, S)$ has property $(*)$ if $S \cap F(G)=1$.

In this note we consider again the case where $S$ is cyclic. We show that $(G, S)$ has property $(*)$ if $F(G)=1$ from which the aforementioned result of T. J. Laffey follows.

Our notation is as in (5). We recall that $F(G)$ denotes the maximal nilpotent normal subgroup of $G$; an $E$-group is the product of a certain set of its subgroups $E_{1}, \ldots, E_{n}$ satisfying $\left[E_{i}, E_{i}\right]=E_{i}, E_{i} / Z\left(E_{i}\right)$ is simple and $\left[E_{i}, E_{j}\right]=1(1 \leqslant i, j \leqslant n, i \neq j)$; and, finally, $F^{*}(G)$ denotes the generalised Fitting subgroup of $G$, namely $F^{*}(G)=F(G) \cdot E(G)$ where $E(G)$ is the (unique) maximal normal $E$-subgroup of $G . F^{*}(G)$ contains its centraliser in $G$ (cf. (1)).

\section{The proof of the theorem}

An error in part III of my original proof was kindly pointed out to me by Thomas J. Laffey together with the idea of the alternative part III given here.

Theorem. Let $G$ be a finite group with $F(G)=1$ and let $S$ be a cyclic subgroup of $G$. Then there exists $x \in G$ such that $S \cap x^{-1} S x=1$.

Proof. By induction on $|G|+|S|$.

(I) We may assume that $|S|$ is square free, i.e. $S=S_{1} \times \cdots \times S_{r}$ with $\left|S_{i}\right|=p_{i}$ prime, $p_{i} \neq p_{j}$ for $i \neq j(1 \leqslant i, j \leqslant r)$ and $r>1$.

Because $S$ is cyclic and so it contains a subgroup $S_{0}=S_{1} \times \cdots \times S_{r}$ as claimed and $S_{0} \cap x^{-1} S_{0} x=1$ if and only if $S \cap x^{-1} S x=1$. If $S_{0} \neq S$ we have 20/3-D 
the result by induction. Also if $r=1$ the result follows from our assumption that $F(G)=1$.

(II) We may assume $G=\left(N_{1} \times \cdots \times N_{t}\right) S$ where $N_{1}, \ldots, N_{t}$ are the minimal normal subgroups of $G$.

Because, since $F(G)=1$, it follows that the generalised Fitting subgroup of $G$ is $N_{1} \times \cdots \times N_{t}$ where $N_{1}, \ldots, N_{t}$ are the minimal normal subgroups of $G$ and by a theorem of Bender (1), $N_{1} \times \cdots \times N_{t}$ contains its centralizer in $G$. We consider $G_{0}=\left(N_{1} \times \cdots \times N_{t}\right) S$. If $S$ has a core $C$ in $G_{0}$ then by (I) we may write $C=C_{1} \times C_{2}$ where $C_{1} \leqslant N_{1} \times \cdots \times N_{t}$ while $C_{2} \cap$ $\left(N_{1} \times \cdots \times N_{t}\right)=1$. But then $C_{2}\left(N_{1} \times \cdots \times N_{t}\right)=C_{2} \times N_{1} \times \cdots \times N_{t}$ contradicting the fact that $N_{1} \times \cdots \times N_{t}$ contains its centralizer. So $C=$ $C_{1} \triangleleft N_{1} \times \cdots \times N_{t}$ and if $c$ is a generator of $C$, say $c=c_{1} \ldots c_{t}$ with $c_{1} \in N_{1}, \ldots, c_{t} \in N_{t}$, and if $x \in N_{1}$ then $c^{x}=c_{1}^{x} \ldots c_{t}^{x}=c_{1}^{x} c_{2} \ldots c_{t}=$ $c_{1}^{s} c_{2}^{s} \ldots c_{1}^{s}$ for some $s \in N$. Thus $c_{1}^{x}=c_{1}^{s}$ and $\left\langle c_{1}\right\rangle \triangleleft N_{1}$ so $c_{1}=1$. Similarly $c_{2}=\cdots=c_{t}=1$. Hence $C=1, S$ has no core in $G_{0}$ and $F\left(G_{0}\right)=1$. So if $|G|+|S|>\left|G_{0}\right|+|S|$ we have the result by induction.

(III) We may assume $t=1$, so that $G=M S$ where $M$ is a minimal normal subgroup of $G$.

Because otherwise, in view of (I) and (II), all the following is welldefined:

(1) $S=C_{1} \times\left(\left(N_{1} \times \cdots \times N_{t}\right) \cap S\right)$.

(2) $C_{2}$ is the product of all the subgroups of prime order of $S$ which are not contained in $C_{1}$, but are contained in $C_{G}\left(N_{2} \times \cdots \times N_{t}\right)$.

(3) $C_{3}$ is the product of all the subgroups of prime order of $S$ which are not contained in $C_{1}$, but are contained in $C_{G}\left(N_{1}\right)$.

(4) If $Q$ is a subgroup of prime order of $S$, but $Q$ is not a subgroup of $C_{1} \times C_{2} \times C_{3}$, then since $Q$ must be contained in $N_{1} \times \cdots \times N_{t}$, we can fix a generator $a_{Q} b_{Q}$ of $Q$ with $a_{Q} \in N_{1}$ and $b_{Q} \in N_{2} \times \cdots \times N_{r}$.

(5) $D$ is the product of all the subgroups $\left\langle a_{Q}\right\rangle$ of $N_{1}$ defined in (4). (4).

(6) $E$ is the product of all the subgroups $\left\langle b_{Q}\right\rangle$ of $N_{2} \times \cdots \times N_{1}$ defined in

(7) $S^{(1)}=C_{1} \times C_{2} \times D ; S^{(2)}=C_{1} \times C_{3} \times E ; \quad G_{1}=S^{(1)} N_{1}$; $G_{2}=S^{(2)}\left(N_{2} \times \cdots \times N_{t}\right)$.

From these definitions we see that $S^{(1)}$ is cyclic of an order dividing $|S|$ and so of square-free order by (I).

If $F_{2}\left(G_{1}\right)$ denotes the inverse image of $F\left(G_{1} / F\left(G_{1}\right)\right)$ in $G_{1}$, then $F_{2}\left(G_{1}\right)$ is a normal solvable subgroup of $G_{1}$ and as such it intersects $N_{1}$ trivially by (II). Thus it is cyclic and therefore equals $F\left(G_{1}\right)$. Hence $G_{1} / F\left(G_{1}\right)$ satisfies the assumption of the theorem and by the induction assumption $S^{(1)} F\left(G_{1}\right) / F\left(G_{1}\right)$ has disjoint conjugates. That is to say $S^{(1)} \cap S^{(1) x} \leqslant F\left(G_{1}\right)$ for some $x \in N_{1}$. But since $F\left(G_{1}\right)$ is cyclic of order dividing $|S|$, this means $S^{(1)} \cap S^{(1) x}$ is normal in $G_{1}$ and any subgroup of $S^{(1)} \cap S^{(1) x}$ is normal in $G_{1}$. 
The argument above applies also to $G_{2}$ and $S^{(2)}$ so that we have:

(8) There are $x \in N_{1}$ and $y \in N_{2} \times \cdots \times N_{1}$ such that any subgroup of $S^{(1)} \cap S^{(1) x}$ is normal in $G_{1}$ and any subgroup of $S^{(2)} \cap S^{(2) y}$ is normal in $G_{2}$.

Let $Q$ be a subgroup of prime order of $S \cap S^{x y^{-1}}$. Then by (I) we have

(9) $Q^{x}=Q^{y}$.

If $Q$ is a subgroup of $C_{1}$ and $s$ is a generator of $Q$, then by (9) $x^{-1} s x=y^{-1} s^{i} y$ for some integer $i \geqslant 1$; but then $[s, x]=s^{i-1}\left[s^{i}, y\right]$ so that $s^{i-1}=[s, x]\left[s^{i}, y\right]^{-1} \in Q \cap G^{\prime}=Q \cap\left(N_{1} \times \cdots \times N_{t}\right)=1$. Thus $i=1$ and $[s, x]=[s, y] \in N_{1} \cap N_{2} \times \cdots \times N_{t}=1$. Therefore $Q=Q^{x}=Q^{y}$ so that $Q \leqslant$ $S^{(1)} \cap S^{(1) x} \cap S^{(2)} \cap S^{(2) y}$ and by (8) $Q \triangleleft G$, a contradiction. If $Q$ is a subgroup of $C_{2}$ then by (2) and (8) $Q=Q^{y}$; by (9) $Q=Q^{x}$; by (7) $Q \leqslant S^{(1)} \cap S^{(1)} x$; by (8) $Q \triangleleft G_{1}$ which together with $Q \leqslant S$ and $Q \leqslant C_{G}\left(N_{2} \times \cdots \times N_{G}\right)$ imply $Q \triangleleft G$ a contradiction.

A similar argument shows $Q$ cannot be a subgroup of $C_{3}$ either, so we have that $Q$ is not a subgroup of $C_{1} \times C_{2} \times C_{3}$ and by (4) and (9) we get $\left(a_{Q} b_{Q}\right)^{x}=\left[\left(a_{Q} b_{Q}\right)^{i}\right]^{y}$ for some integer $i \geqslant 1$; but then by (5), (6) and (8) we get $\left\langle a_{Q}\right\rangle^{x}=\left\langle a_{Q}\right\rangle \leqslant D \cap D^{x} \leqslant S^{(1)} \cap S^{(1) x}$ so $\left\langle a_{Q}\right\rangle \triangleleft G_{1}$ by (8) and $N_{1}$ contain a normal abelian subgroup, a contradiction unless $a_{Q}=1$, in which case the same argument shows $\left\langle b_{Q}\right\rangle^{y}=\left\langle b_{Q}\right\rangle$ is a normal abelian subgroup of $N_{2} \times$ $\cdots \times N_{t}$ again a contradiction. Thus $S \cap S^{x y-1}=1$ and the assertion (III) is proved.

(IV) We now get the final contradiction as follows: Since $S$ has no conjugate which intersects it trivially then if $M$ is as in (III), we have $M=\bigcup_{i=1}^{r} N_{M}\left(S_{i}\right)$ where the $S_{i}$ are as in (I). Let the notation be chosen so that $n_{0}=\left|M: N_{M}\left(S_{1}\right)\right| \leqslant\left|M: N_{M}\left(S_{i}\right)\right|$ for $i=2, \ldots, r$. Then

$$
|M| \leqslant \sum_{i=1}^{r}\left|N_{M}\left(S_{i}\right)\right|=\sum_{i=1}^{r} \frac{|M|}{\left|M: N_{M}\left(S_{i}\right)\right|} \leqslant \frac{r|M|}{\left|M: N_{M}\left(S_{1}\right)\right|}
$$

and it follows that $n_{0} \leqslant r$.

Let $p$ be the maximum prime divisor of $|S|$. Then by (I), $r<p$ and so $n_{0}<p$.

Now if $n_{1}$ is the number of conjugates of $S_{1}$ in $G$ then $n_{1}=\left|G: N_{G}\left(S_{1}\right)\right|$ and by (III) $N_{G}\left(S_{1}\right)=N_{M}\left(S_{1}\right) \cdot S$ so that

$$
n_{1}=\frac{|G|}{\left|N_{G}\left(S_{1}\right)\right|}=\frac{|M||S|\left|N_{M}\left(S_{1}\right) \cap S\right|}{|M \cap S|\left|N_{M}\left(S_{1}\right)\right||S|} \leqslant \frac{|M|}{\left|N_{M}\left(S_{1}\right)\right|}=n_{0} .
$$

It follows that $n_{1}<p$. But $G$ acts by conjugation on the $n_{1}$ conjugates of $S_{1}$ and since $p \mid G$ but $p \nmid n_{1}$ !, this action has a non-trivial kernel $N ; N \cap M$ is a normal subgroup of $G$; so by (III) $N \cap M=1$ in which case $N$ centralises $M$ against the proof of (II), or $N \cap M=M$ and so $M$ normalises $S_{1}, S_{1} \triangleleft G$ against our assumption $F(G)=1$. 


\section{REFERENCES}

(1) H. Bender, On Groups with Abelian Sylow 2-Subgroups, Math. Z., 117 (1970), 164-176.

(2) J. S. BroadkeY, A Note on Finite Groups with an Abelian Sylow Subgroup, Proc. Amer. Math. Soc., 14 (1963), 132-133.

(3) M. Herzog, Intersection of Nilpotent Hall Subgroups, Pacific J. Math., 36 (1971), 331-333.

(4) N. ITO, Über den kleinsten p-Durchschnitt auflösbarer Gruppen, Arch. Math. (Basel) 9 (1958), 27-32.

(5) T. J. LAfFey, A Problem on Cyclic Subgroups of Finite Groups, Proc. Edinburgh Math. Soc., (2) 18 (1973), 247-249.

University of Illinois at Chicago Circle Mathematics Department

P.O. Box 4348

Chicago, Illinois 60680

U.S.A. 